\section{RTh 07}

SYMPTOMATIC RADIOTHERAPY OF BONE METASTASES H.J.Tkocz, K. Schnabel, W. Berber ich, M.Niewa Id, U. Unlmann and B.Scharding

In a pilotstudy 91 pat. with bone infiltration or metastases of various primary tumours were treated. Irradiation was carried out using photons or fast electrons. Five dosage and fractionation schemes were used: 1. 40 Gy in 20 fract. oy. 4 weeks ( 49 cases); 2.30 Gy in 15 fract. ov. 3 weeks (6 cases); 3.20 gy in 5 fract. ov. 1 week (20 cases); 4.20 Gy in 5 fract. ov. 1 week +10 Gy in 5 fract. ov. 1 week ( 10 cases); 5. 50-60 Gy in 25-30 fract. ov. 5-6 weeks (6 cases). Account was taken of the degree and length of páin reduction, the general condition, restoration of mobility or ability to walk and recalcification. 71 patients $(78 \%)$ with an average survival time of 38 weeks showed a positive reaction to the irradiation. The patients treated according to scheme $\mathrm{Nr} .1$ experienced in $46 / 49$ cases (94\%) relief of their complaints, lasting on average 25 weeks. The 6 pat. treated only with. 30 Gy (scheme $\mathrm{Nr}$. 2) all experienced pain-relief for approx. 28 weeks. In $80 \%(16)$ of the cases treated according to scheme 3 a palliative effect was observed, but only of 8 weeks duration. The further application of 10 Gy in 5 fractions (scheme 4) did not increase the pall. effect: in 6 of 10 cases $(60 \%)$ pain-relief for an average of 13 weeks was achieved. The response-rate $(100 \%)$ of the 6 pat. treated with 50-60 Gy and the average duration of remission of pain (24 weeks) are comparable with those of group 1 and 2 .

Summarising, the best palliative effects were achieved using schemes 1 and 2 .

Abteilung für Strahlentherapie, Radiologische Universitätsklinik D-6650 Homburg/Saar

\section{RTh 08}

SECONDARY MAL IGNANCIES AFTER RADIOTHERAPY: A RETROSPECTIVE ANALYSIS OF 454 CASES

R. Fietkau, L. Stehr, T.L. Diepgen, R. Sauer

The knowledge of radiation induced malignancies is mostly based only on case reports. We analyzed the date of 98 case reports (1902-1984) including 454 secondary malignancies (52\% sarcomas, 38\% carcinomas, 8,5\% leukemias, two cases of brain tumours) after radiotherapy.

Sarcomas occured after irradiation with a dose of 12-70Gy appearing more frequently after radiotherapy of malignant diseases (64\%) than after radiotherapy of benign diseases (41\%).

Carcinomas were mostly seen after irradiation with doses up to 30 Gy occuring more frequently after irradiation of benign diseases (57\%) than of malignant diseases (30,7\%). More carcinomas were obsefyed after conventional irradiation (40\%) than after ${ }^{6}$ Co irradiation (25\%). The percentage of thyroid carcinomas was remarkably high (60\% of carcinomas), if the thyroid irradiation took place during childhood.

The occurance of leukemias did not show any dose-relation. They appeared mainly after irradiation of malignant diseases (15\%) than in case of benign diseases $(2,7 \%)$. If radiotherapy was combined or followed by chemotherapy the percentage increased up to $42 \%$ The mean interval until diagnosis of the secondary malignancy varied between 7,1 years (leukemias), 12, 1 years (sarcomas) and 19 years (carcinomas). Either additional chemotherapy as surgery as well reduced generally the interval: from 15,7 to 7,4 years (chemotherapy); from 18,7 to 11,4 years (surgery).

Strahlentherapeutische Klinik und Poliklinik Universität Erlangen-Nürnberg Universitätsstr. 27, D - 8520 Erlangen

\section{NCy 01}

TREATMENT OF SOLOID TUMORS AND ACUTE LEUKEMTAS WITH THE POLYAMINE ANTIMETABOLITES DIFLUOROMETHYLORNITHINE AND METHYLGLYOXAL BIS (GUANYJHYDRAZONE)

M. Garbrecht, S. Busch, T. Iangenbuch and G. Ziemer

A clinical phase II study was initiated to evaluate the efficacy of combination treatment with the polyamine antimetabolites $\alpha$-difluoromethylornithine(DEMO) and methylglyoxal bis (guanylhydrazone) (MGBG) . Synergism of DFMO and MGBG in inhibiting tumor cell growth has been proven in different animal tumor cell systems (Biochem.Biophys. Acta 674,169-177(1981); Cancer 53,1294-1298(1984).According to these data we initiated a clinical phase II study to evaluate the efficacy of DFNO+MGBG in patients (pts) with malignant melanoma, hypernephroma, testicular tumors, large bowel cancer, esophagus carcinoma and acute leukemias.All pts had received intensive prior treatment and showed progressive disease. 16 pts are evaluable. 3 pts had to stop DFMO-MGBG therapy because of severe side effects (myelosuprresion 2, severe mucositis 1). 6 pts responded with partial remission (1-12month), 4 pts showed stable disease (1-7+month) . 6 pts did not respond. Partial remission or long term stable disease was seen in all of 5 pts with disseminated hypemephroma. Partial remission was also seen in 1 pt with AMML, 1 pt with esophagus carcinoma, 1 pt with melanoma and in 2 pts with large bowel cancer. Monitoring of polyamine excretion in the urine of pts revealed no significant reduction of putrescine. This might be due to interconversion of spermine and spermidine to putrescine as well as liberation of putrescine from necrotzing tumor. The fact that 10 pts responded with partial remission or showed long term stable disease despite poor prognosis and heavy pretreatment reconmends further clinical investigation of the DFMO-MGBG combination chemotherapy.

Medizinische Universitätsklinik Hamburg, Abteilung Onkologie - Hämatologie, Martinistraße 52 - D-2000 Hamburg 20

\section{NCy 02}

DECREASED CARDIOIOXICITY OF AIRTAMYCIN BY VERAPAMII

U.Miillerleile,M.Garbrecht,P.,Hanrath, K.Bieber,V.Siglow, D.K.Hossfeld Cardioniyopathy remains a problem in chemotherapy with Adrianycin(ADM) and other Anthracyclins. One out of several hypothetical ways to prevent ADM-cardiomyopathy is the use of calcium entry blockers such as Verapamil. Preliminary data of a pilot study which suggested benefit from the use of Verapemil as a 'protective' agent are now updated on the basis of a greater nunber of patients and an extended program of invasive and non-invasive investigations. The parameters of serial echocardiographical measurenents of left ventricular pump function (shortening fraction:FS, peak fiber shortening velocity:V cf max) in patients treated with ADM and Verapamil (v) $3 \times 40 \mathrm{mg}$ daily were compared with those of pts treated without $V$. The $V$ group includes 35 , the control group 61 pts. In the $V$ group FS and V cf did not decline in the mean: d FS was + 0,026 FS\%/100mgADM/sqm, d Vcf +0,0246 circ/ $\mathrm{sec} / 100 \mathrm{mgADM} / \mathrm{sqm}$ whereas in the control group in the mean d FS and d Vcf showed a clear deterioration by $-3,4 \mathrm{FS} \%$ and $-0,33 \mathrm{circ} / \mathrm{sec}$ per $100 \mathrm{mg} \operatorname{AIM} / \operatorname{sqm}(\mathrm{p}: 0,001)$. Additional radionuclidventriculograms proved good correlation with the echocardiographic data. In 4 pts with cumulative doses of ADM beyond $550 \mathrm{mg} / \mathrm{sqm}$ endonyocardial biopsies: were performed. The biopsy scores(according to Billingham classification) were $0,0,1,1$ with FS rates at the time of biopsy of $34,34,30$,$30 \%$ respectively. One of these pts subsequently developped clinically apparent cardiomyopathy under therapy with Mitoxantron. Phannacocinetic studies in some of the pts showed that $V 3 \times 40 \mathrm{mg}$ daily did not change AIM disposition. The bioavailability of orally administered V however differs remarkably between individual pts.

The conclusions from the present data of this study are:

(1)V probably prevents or delays myocardial damage caused by ADM. (2)The protective effect is presumably caused by prevention of cardionyocyte calcium overload - an event which on the other hand is not specific for ADM-cardionyopathy.

(3)It seems questionable if $3 \times 40 \mathrm{mg} V$ daily is sufficient in all pts to achieve 'protective' serum levels.

(4)Further investigations should be focused on the histopathological evidence of the postulated myocardiac protection and on pharmacocinetic studies of V,ADM and their possible interaction. 